\title{
TWO NUMERICAL SOLUTIONS FOR SOLVING A MATHEMATICAL MODEL OF THE AVASCULAR TUMOR GROWTH
}

\section{Sila Ovgu KORKUT'1,2, Neslisah IMAMOGLU KARABAS ${ }^{3}$, Yasemin BASBINAR $^{2}$}

${ }^{1}$ Izmir Katip Celebi University, Department of Applied Mathematics and Statistics, Izmir, Turkey.

2 Dokuz Eylul University, Institute of Oncology, Department of Translational Oncology, Izmir, Turkey.

${ }^{3}$ Izmir Institute of High Technology, Department of Mathematics, Izmir, Turkey.

Address for Correspondence: Sıla Ovgu KORKUT, E-mail: silaovgu.korkut@ikcu.edu.tr

Received: 28.06.2021; Accepted: 16.08.2021; Available Online Date: 20.09.2021

(C) Copyright 2021 by Dokuz Eylül University, Institute of Health Sciences - Available online at https://dergipark.org.tr/en/pub/jbachs

Cite this article as: Korkut SO, Karabas Neslisah I, Basbinar Y. Two Numerical Solutions for Solving a Mathematical Model of the Avascular Tumor Growth. J Basic Clin Health Sci 2021; 3: 156- 164.

\begin{abstract}
Objective: Cancer which is one of the most challenging health problems overall the world is composed of various processes: tumorigenesis, angiogenesis, and metastasis. Attempting to understand the truth behind this complicated disease is one of the common objectives of many experts and researchers from different fields. To provide deeper insights any prognostic and/or diagnostic scientific contribution to this topic is so crucial. In this study, the avascular tumor growth model which is the earliest stage of tumor growth is taken into account from a mathematical point of view. The main aim is to solve the mathematical model of avascular tumor growth numerically.

Methods: This study has focused on the numerical solution of the continuum mathematical model of the avascular tumor growth described by Sharrett and Chaplin. Unlike the existing recent literature, the study has focused on the methods for the temporal domain. To obtain the numerical schemes the central difference method has been used in the spatial coordinates. This discretization technique has reduced the main partial differential equation into an ordinary differential equation which will be solved successively by two alternative techniques: the 4th order Runge-Kutta method (RK4) and the three-stage strongly-stability preserving Runge-Kutta method (SSP-RK3).

Results: The model has been solved by the proposed methods. The numerical results are discussed in both mathematical and biological angles. The biological compatibility of the methods is depicted in various figures. Besides biological outputs, the accuracies of the methods have been listed from a mathematical point of view. Furthermore, the rate of convergence of the proposed methods has also been discussed computationally.

Conclusion: All recorded results are evidence that the proposed schemes are applicable for solving such models. Moreover, all exhibited figures have proved the biological compatibility of the methods. It is observed that the quiescent cells which are one of the most mysterious cells in clinics tend to become proliferative for the selected parameters.

Keywords: Avascular tumor growth, numerical simulation, mathematical biology, three-stage stronglystability preserving Runge-Kutta method, 4th-order Runge-Kutta Method.
\end{abstract}

\section{INTRODUCTION}

On a cellular scale, cancer is formed by transforming normal cells into abnormal cells which are called tumorigenesis. In other words, cancer basically occurs by the collapse of the perfectly functioning mechanism due to alterations or mutations that 
occurred in DNA. Even though the exact cause of these alterations and/or mutations are, in general, because of heritable and/or environmental, it is still unknown. Moreover, the mutations can also vary by the located tissue and the propagation rate of the disease can vary individually. These characteristics make the disease more complicated. Besides all these facts, the disease is, furthermore, one of the most threatening diseases globally due to its mortality and morbidity rates. Therefore, cancer has a great deal of importance among many researchers not only from medicine or biology but also from applied sciences as well as governments.

The stages of tumor growth are avascular, angiogenesis, and metastatic. That is, all tumors are mainly formed by an avascular phase of growth. The transition of the process can be summarized as follows: a small (avascular) tumor growth is originated from the uncontrolled cell division. As long as it is not supported by the angiogenesis or it is not intervened by the immune system, the avascular tumor growth will be inactive, (1). Otherwise, it continues to expand up to a small size due to inadequate nutrients and oxygen. However, likewise the normal cells, tumor cells also need surrounding tissue for supplying nutrients and oxygen by diffusion, $(2,3)$. Thus, tumor cells stimulate the blood vessels by signals, and angiogenesis, one of the hallmarks of tumor progression, has begun. The vascularization of the host tissue helps to feed it and to increase its size rapidly. Thus, tumors are capable to invade the tissue and spreading to another tissue when the tumor is malignant. This means that the tumor is called a metastatic phase which makes cancer fatal.

Due to the compelling characteristics of cancer, any theoretical, experimentally, or clinically research plays a key role to understand the dynamics of cancer cells and their interactions. Thus, studying this subject is an interest not just in biology or medicine but in applied sciences, as well. Of course, as a guide, both experimental and clinical studies have much attention and impact in medicine. However, both studies have their own challenges. In experimental studies, on one hand, biological variations can affect the results. Moreover, laboratory experiments need enormous costs. Clinical studies, on the other hand, take a very long time to provide reliable and meaningful data. All the mentioned aspects are biological. However, in-silico studies can be used to enrich a deep understanding of such compelling disease and to make predictions and, provide insights and strategies to treatment of this disease. A mathematical model of a biological system or a complex disease is a powerful tool and cheap for the prognosis. Undoubtedly that each disease has its own particular characteristics thus some assumptions can be provided to address real-life situations. It is worth noting that mathematical models can vary for each circumstance and/or assumption.

From a clinical point of view, indeed, modeling the vascular or metastatic tumor growth is more realistic and is, furthermore, vital for clinical treatment. However, a model such a complex system can lead to the loss of its reliability. Thus, studying much simpler models like avascular tumor growth can offer a deeper perspective and can be more wisely. Moreover, avascular tumors can be produced easily and cheaply in-vitro multicellular spheroids which include in-vivo characteristics such as proliferation or nutrient gradients as well as the tumor microenvironment, (4). To date, a lot of effort has been invested into developing a mathematical model for avascular tumor growth. The earliest model of avascular tumor growth was originated in 1955 by Thomlinson and Gray (5). In the study of Burton, (6), the growth of solid tumors is considered as a diffusion equation. Greenspan has observed an annulus of quiescent cells between proliferative and necrotic cells in the multicellular spheroids, (7). Greenspan's study is accepted as the fundamental of mathematical oncology. Inspired by (7), many remarkable studies have been done, (8-12). The authors promote the interested reader on mathematical models of the avascular tumor to the descriptive review in (13). Additionally, one can be referred to (14) for a brief introduction to mathematical oncology.

Of course, proposing an appropriate solution which is also physically compatible is as crucial as modeling the situation mathematically. Mathematical models can be categorized as continuum models, discrete models, and hybrid. There are several studies on the numerical solutions of the continuum avascular tumor growth in the literature. For instance, conservative Galerkin characteristics algorithm combining with the trapezium integration has been applied to the avascular tumor growth model in (15), a numerical solution based on forward time-central space discretization has been studied for the case of hypoxic cell regulation in avascular tumor growth in (16). A couple of nonlinear partial differential equations via parallel programming in (17). More recently, an adaptive mesh method called moving 
mesh combining with implicit Euler method is applied to an avascular tumor growth model in (18). Moreover, another application of the moving mesh method combined with forward time discretization has been proposed in (19) to solve Sherratt and Chaplin's model of avascular tumor growth. Furthermore, avascular tumor growth using Gompertz growth rate has been approximated by implementing a finite difference method in (20).

Our main aim is not to underestimate the models or methods which are not our consideration in this study but to propose alternative numerical methods for solving such a continuum avascular tumor growth. To do this, the model developed by Sherratt and Chaplin, (11), has been taken into account. Central difference approximation is used for the spatial domain and the temporal domain is solved not only by the 4th order Runge-Kutta method (RK4) but also three-stage strongly-stability preserving Runge-Kutta method (SSP-RK3). To the best of the authors' knowledge, both RK4 and SSP-RK3, (21), are the first time applied to solve the specified problem.

Under the light of the knowledge mentioned above, the organization of the present study is as follows: Section 2 describes the mathematical model and the numerical schemes considered in this study. Results and discussions have been provided in Section 3 which is followed by the Conclusion of the study.

\section{MATERIAL AND METHODS Mathematical Model}

As highlighted earlier Sherratt and Chaplin's mathematical model for the avascular tumor has been taken into account. The main focus of the current study is to suggest some alternative techniques for solving the avascular tumor growth model, numerically. To do so, a thin disc-shaped domain, that is one-dimensional spatial coordinates, has been considered. It is important to emphasize that this is the most realistic model of tumor growth, conversely, two- or three-dimensional models are more realistic. However, studying with a simple form of the equation is much understandable if the study is descriptive.

The specified model is as follows:

$$
\begin{aligned}
& \frac{\partial p}{\partial t}=\frac{\partial}{\partial x}\left(\frac{p}{p+q} \frac{\partial(p+q)}{\partial x}\right)+g(c) p(1-p-q- \\
& n)-f(c) p, \quad x \in\left[x_{0}, x_{\text {end }}\right], \quad t>0, \\
& \frac{\partial q}{\partial t}=\frac{\partial}{\partial x}\left(\frac{q}{p+q} \frac{\partial(p+q)}{\partial x}\right)+f(c) p- \\
& h(c) q, \quad x \in\left[x_{0}, x_{\text {end }}\right], \quad t>0,
\end{aligned}
$$

$\frac{\partial n}{\partial t}=h(c) q, \quad x \in\left[x_{0}, x_{\text {end }}\right], t>0$,

where $p(x, t), q(x, t)$ and $n(x, t)$ represent the density of proliferating cells, quiescent cells, and necrotic cells. Notice that $\frac{p}{p+q} \frac{\partial(p+q)}{\partial x}$ and $\frac{q}{p+q} \frac{\partial(p+q)}{\partial x}$ denote the migration of the cells from the dense medium to the less dense medium. Moreover, $g(c)$ is the cell mitosis and $f(c)$ denotes the rate of the proliferative cells turning into quiescent phase while the quiescent cells becoming necrosis at the rate $h(c)$. Furthermore, $c(x, t)$ stands for the local nutrient concentration which is taken as

$$
c=\frac{c_{0} \gamma}{\gamma+p}(1-\alpha(p+q+n))
$$

where $\gamma$ and $\alpha$ are some scalars. On the other hand, $c_{0}$ is the initial nutrient concentration. It is important to note that $p, q$, and $n$ are functions of $x$ and $t$. Here, $x$ and $t$ stand for the location and time variables, respectively. For more detailed discussions the authors refer the reader to $(9,15)$. It is also noted that both $f(c)=0$, and $h(c)=0$ as $c \rightarrow \infty$ where $f(c)>$ $h(c),(13)$. This means also that the functions $f$ and $h$ are decreasing functions, $g(c)$ is, on the contrary, increasing.

For the sake of clarity of notations let $r=p+q, M_{p}=$ $\frac{p}{r}$ and $M_{q}=\frac{q}{r}$. With the help of chain rule the model in Equations (1)-(3) can be expressed more explicitly as follows:

$$
\begin{aligned}
& \frac{\partial p}{\partial t}=\left(M_{p}\right)_{x} \frac{\partial r}{\partial x}+M_{p} \frac{\partial^{2} r}{\partial x^{2}}+g(c) p(1-p-q- \\
& n)-f(c) p, \quad x \in\left[x_{0}, x_{\text {end }}\right], \quad t>0, \\
& \frac{\partial q}{\partial t}=\left(M_{q}\right)_{x} \frac{\partial r}{\partial x}+M_{q} \frac{\partial^{2} r}{\partial x^{2}}+f(c) p- \\
& h(c) q, \quad x \in\left[x_{0}, x_{\text {end }}\right], \quad t>0, \\
& \frac{\partial n}{\partial t}=h(c) q, \quad x \in\left[x_{0}, x_{\text {end }}\right], \quad t>0,
\end{aligned}
$$

where

$$
\left(M_{p}\right)_{x}=\frac{p_{x} r-r_{x} p}{r^{2}} \text { and }\left(M_{q}\right)_{x}=\frac{q_{x} r-r_{x} q}{r^{2}}
$$

Throughout the study, the specified model subject to the zero-flux condition, that is Neumann-type boundary conditions. More precisely, $\frac{\partial p}{\partial x}=\frac{\partial q}{\partial x}=\frac{\partial n}{\partial x}=$ 0 at $x=x_{0}$ and $x=x_{\text {end }}$. Moreover, the initial conditions are taken as follows:

$$
p(x, 0)=e^{-0.1 x}, \quad q(x, 0)=0, \quad n(x, t)=0 \quad \text { and } \quad c_{0}=1 .
$$


Due to the inability of the computers, $x_{\text {end }}$ cannot be taken as $\infty$. Thus, for a more realistic domain $x_{\text {end }}$ is chosen sufficiently large, that is $x_{\text {end }}=210$.

\section{Numerical Scheme}

\section{Spatial Discretization}

The specified model in Equation (5) corresponds to a system of the coupled nonlinear partial differential equation (PDE). Finite difference methods are the most preferred approach for solving such a system. However, in this study, central difference approximation is utilized for discretizing the spatial coordinates of Equation (5). In so doing, the system of PDE reduces into a system of the ordinary differential equation (ODE) which can be solved by a higher-order method, successively.

Let $\Delta x=x_{i+1}-x_{i}$ for $i=0,1 \ldots, N_{x}$ where $N_{x}$ is the number of divisions. Then, one can obtain

$$
\begin{aligned}
& \dot{p}_{i}=M_{i} \frac{r_{i+1}-r_{i-1}}{2 \Delta x}+\frac{p_{i}}{r_{i}} \frac{r_{i+1}-2 r_{i}+r_{i-1}}{\Delta x^{2}}+ \\
& g\left(c_{i}\right) p_{i}\left(1-p_{i}-q_{i}-n_{i}\right)-f\left(c_{i}\right) p_{i}, \\
& \dot{q}_{i}=N_{i} \frac{r_{i+1}-r_{i-1}}{2 \Delta x}+\frac{q_{i}}{r_{i}} \frac{r_{i+1}-2 r_{i}+r_{i-1}}{\Delta x^{2}} \\
& \quad+f\left(c_{i}\right) p_{i}-h\left(c_{i}\right) q_{i}, \\
& \dot{n}_{i}=h\left(c_{i}\right) q_{i},
\end{aligned}
$$

Notice that $p_{i}=p\left(x_{i}, t\right), q_{i}=q\left(x_{i}, t\right)$, and $n_{i}=n\left(x_{i}, t\right)$. Moreover, $\dot{p}_{i}, \dot{q}_{i}$, and $\dot{n}_{i}$ represent the derivatives of $p, q$, and $n$ with respect to $t$ at $x=x_{i}$.

$$
M_{i}=\frac{\frac{p_{i+1}-p_{i-1}}{2 \Delta x} r_{i}-p_{i} \frac{r_{i+1}-r_{i-1}}{2 \Delta x}}{r^{2}} \text { and } \quad N_{i}=\frac{\frac{q_{i+1}-q_{i-1}}{2 \Delta x} r_{i}-q_{i} \frac{r_{i+1}-r_{i-1}}{2 \Delta x}}{r^{2}}
$$

After employing the discretization algorithm and imposing the boundary conditions the differentiation matrices are obtained as follows:

$$
D_{1}=\frac{1}{2 \Delta x}\left[\begin{array}{ccccc}
0 & 0 & 0 & \cdots & 0 \\
-1 & 0 & 1 & \ddots & \vdots \\
0 & \ddots & \ddots & \ddots & 0 \\
\vdots & \ddots & -1 & 0 & 1 \\
0 & \cdots & 0 & 0 & 0
\end{array}\right] \text { and } D_{2}=\frac{1}{\Delta x^{2}}\left[\begin{array}{ccccc}
-2 & 2 & 0 & \cdots & 0 \\
1 & -2 & 1 & \ddots & \vdots \\
0 & \ddots & \ddots & \ddots & 0 \\
\vdots & \ddots & 1 & -2 & 1 \\
0 & \cdots & 0 & 2 & -2
\end{array}\right]
$$

where $D_{1}$ and $D_{2}$ are $\left(N_{x}+1\right) \times\left(N_{x}+1\right)$ sparse matrices. Thus, the attained system of ODEs in Equations (7)-(9) can be expressed simply in a matrix-vector form as follows:

$$
\left[\begin{array}{l}
\dot{p}(x, t) \\
\dot{q}(x, t) \\
\dot{n}(x, t)
\end{array}\right]=\left[\begin{array}{l}
F_{p}(p, q, n) \\
F_{q}(p, q, n) \\
F_{n}(p, q, n)
\end{array}\right]
$$

where $x$ stands for the vector form of the discretized domain and

$F_{p}(p, q, n)=M D_{1} r+\frac{p}{r} D_{2} r+g(c)(1-p-q-n)-$

$f(c) p$,

$F_{q}(p, q, n)=N D_{1} r+\frac{q}{r} D_{2} r+f(c) p-h(c) q$,

$F_{n}(p, q, n)=h(c) q$.

Notice that $M=\frac{D_{1} p r-p D_{1} r}{r^{2}}$ and $N=\frac{D_{1} q r-q D_{1} r}{r^{2}}$.

\section{Temporal Integration}

The main objective of this section is to share the two alternative methods in a brief and understandable way with the readers. To do so, we first recall Equation (13) by making the notations simpler such that

$$
\begin{aligned}
& \dot{V}(x, t)=G(x, t, V) \\
& V(x, 0)=V_{0}(x) .
\end{aligned}
$$

Notice that $V(x, t)=\left[\begin{array}{lll}p(x, t) & q(x, t) & n(x, t)\end{array}\right]^{T}$ and $G(x, t, V)=\left[F_{p}(p, q, n) \quad F_{q}(p, q, n) \quad F_{n}(p, q, n)\right]^{T}$ where $T$ stands for the transpose of a vector. Moreover, $\dot{V}(x, t)$ represents $\frac{\partial}{\partial t} V(x, t)$.

In this study, two alternatives already exist methods have been suggested to the literature. This is not to say that this study is not novel, on the contrary, we have never encountered such a study in the literature survey we have done so far. The methods discussed throughout the current study are RK4 and SSP-RK3 which are described as follows:

$$
\mathrm{RK} 4\left\{\begin{array}{l}
k_{1}=G\left(x, t_{n}, V_{n}\right) \\
k_{2}=G\left(x, t_{n}+\frac{\Delta t}{2}, V_{n}+\Delta t \frac{k_{1}}{2}\right) \\
k_{3}=G\left(x, t_{n}+\frac{\Delta t}{2}, V_{n}+\Delta t \frac{k_{2}}{2}\right) \\
k_{4}=G\left(x, t_{n}+\Delta t, V_{n}+\Delta t k_{3}\right) \\
V_{n+1}=V_{n}+\frac{\Delta t}{6}\left(k_{1}+2 k_{2}+2 k_{3}+k_{4}\right)
\end{array}\right.
$$

and

$$
\text { SSP - RK3 }\left\{\begin{array}{l}
k_{1}=G\left(x, t_{n}, V_{n}\right) \\
k_{2}=\frac{3}{4} G\left(x, t_{n}, V_{n}\right)+\frac{1}{4} \Delta t\left(k_{1}+G\left(x, t_{n}+\Delta t, k_{1}\right)\right) \\
V_{n+1}=\frac{1}{3} V_{n}+\frac{2}{3} k_{2}+\frac{2}{3} \Delta t G\left(x, t_{n}+\frac{\Delta t}{2}, k_{2}\right)
\end{array}\right)
$$

It is noted that $\Delta t$ is the time step, that is $\Delta t=\frac{t_{\text {end }}}{N_{t}}$ where $N_{t}$ is the number of divisions in time and that $n$ stands for the iteration of time for $n=0,1, \ldots, N_{t}$. It is 


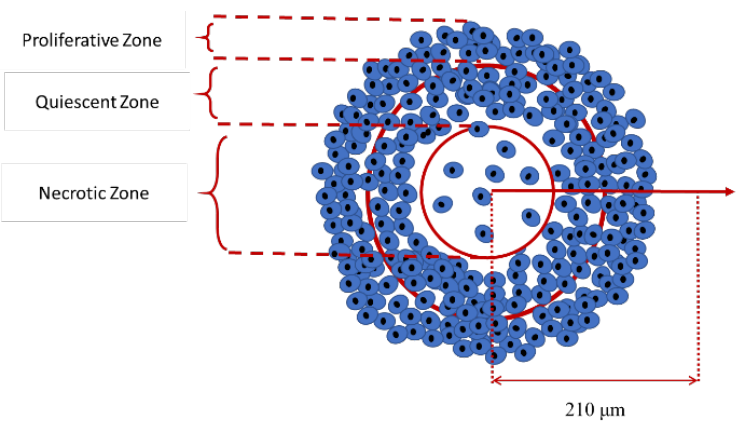

Figure 1. Schematic illustration of the avascular tumor.

important to emphasize that SSP-RK3 is also known as third-order total variation diminishing the RungeKutta method in literature. For a detailed discussion on SSP-RK3, the authors refer the reader to (21).

\section{RESULTS AND DISCUSSION}

Obtaining a numerical solution for nonlinear partial differential equations is challenging, especially if the phenomena preserve some characteristics. Even though we assure theoretically that the numerical method will eventually converge to the exact solution, some problems can occur computationally such as stability. Thus, any numerical methods should be confirmed from a computational point of view. This section is dedicated to present the results of the performed numerical schemes on the addressed avascular tumor growth model. The model parameters are chosen as follows:

$$
\begin{aligned}
& f(c)=\frac{1-\tanh (4 c-2)}{2}, \\
& g(c)=\beta e^{\beta c} \\
& h(c)=f(c) / 2 .
\end{aligned}
$$

Notice that the proliferation rate is considered as Gompertz's growth rate. All computations have been executed on Intel Core i7-6700HQ 2.60Ghz and $16 \mathrm{~GB}$ of RAM and implemented via the MATLAB2018b programming language.

Before presenting the numerical results the biological illustration expressing the avascular tumor cell has been depicted in Figure 1. From a biological angle, the avascular tumor model has three main zones: necrotic zone, quiescent zone, and proliferative zone. In the spirit of the proliferative zone, the tumor cells tend to proliferate and grow whereas the cells tend to die due to the lack of nutrients and oxygen in the

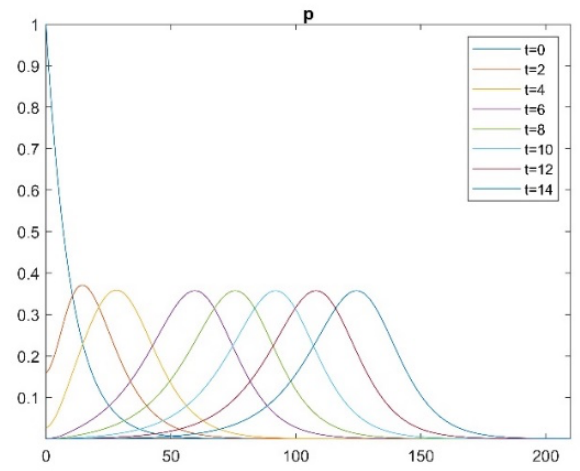

(a)

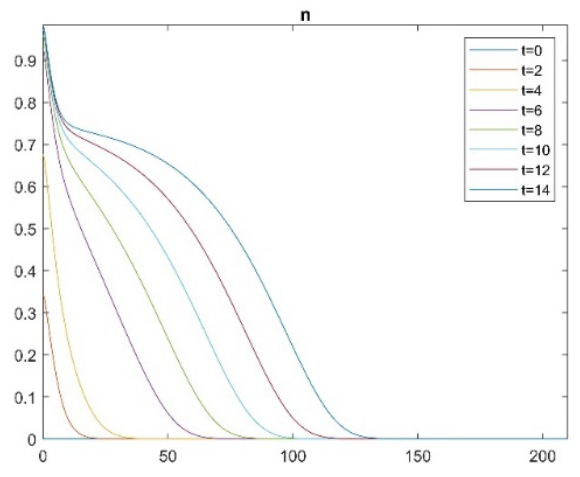

(c)

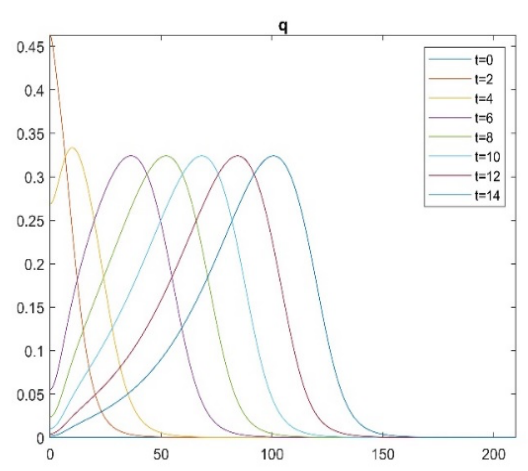

(b)

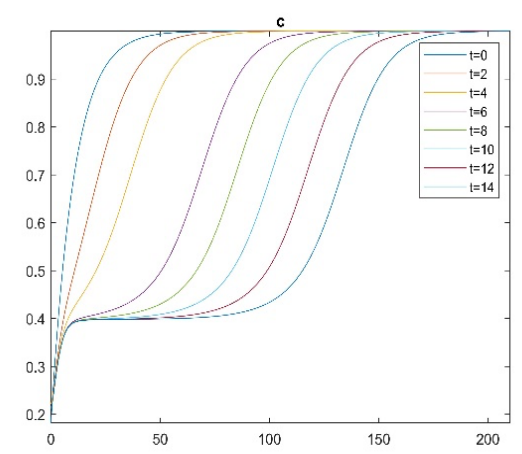

(d)

Figure 2. The densities of proliferating (a), quiescent (b), and the necrotic cell (c) for $\alpha=0.8$. The obtained results belong to RK4. 


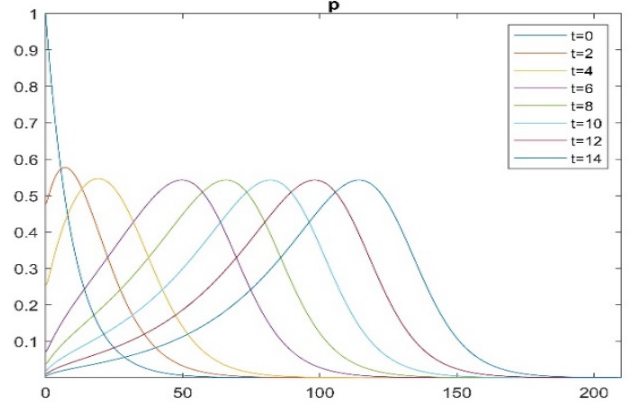

(a)

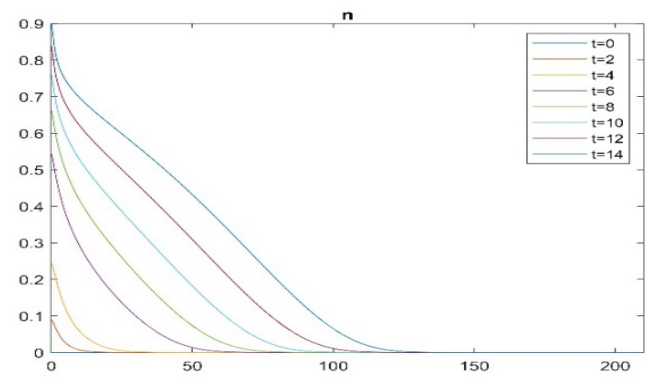

(c)

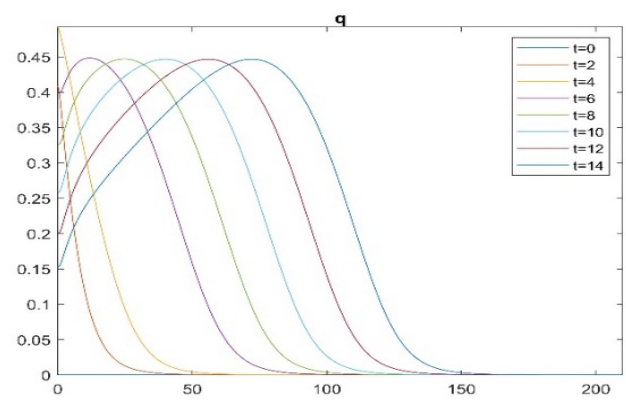

(b)

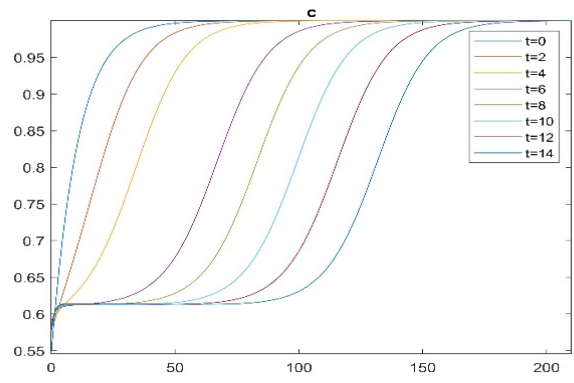

(d)

Figure 3. The densities of proliferating (a), quiescent (b), and the necrotic cell (c) obtained by RK4 for $\alpha=0.4$.

necrotic zone. However, cells in the quiescent zone are mysterious. Therefore, quiescent cells play a vital role in the phenotype of the tumor.

Figure 2 illustrates the density of the proliferating, quiescent, and necrotic cells in $t=0,2, \cdots, 14$ weeks computed by RK4. For the exhibited figure, Figure 1, the constant parameters are taken as $\alpha=0.8, \beta=0.5$ and $y=10$. Moreover, the domain $(0,210)$ is divided into 420 nodes with mesh size $\Delta x=0.5$ and the time step is considered as $\Delta t=0.1 \approx 16.8$ hours. All figures are compatible with both the literature, (19-20), and the biological processes.

Moreover, Figure 3 shows the significance of the $\alpha$ parameter on the solutions. One can be observed that the smaller values of $\alpha$ the proliferating rate is bigger. The density of proliferating cells, therefore, is more than those of $\alpha=0.8$. Moreover, for the smaller choice of $\alpha$, the necrotic core is more linear, that is the necrotic cells tend to 0 faster.

Likewise, the density of the proliferating, quiescent, and necrotic cells in $t=0,2, \cdots, 14$ weeks has been obtained by SSP-RK3. Similar to RK4, in Figure 4, the numerical results are attained for the choice of $\alpha=0.8, \beta=0.5, y=10$ where $\Delta x=0.5$ and $\Delta t=0.1 \approx 16.8$ hours. The exhibited figures, Figure 4 and Figure 5 , are in good agreement with those in the literature. Besides Figure 4, Figure 5 describes the behavior of the SSP-RK3 when $\alpha=0.4$. Similar reactions of those for RK4 are observed for SSP-RK3.

Due to the similar behavior of the numerical solutions, SSP-RK3 also provides biological compatibility. One can observe from Figures 2-5 that as time passes the proliferating cells tend to migrate toward the outer edge. Toward the outer edge, the density of the proliferating cells is much more than those of quiescent and necrotic. Additionally, the necrotic cells decrease moving away from the tumor center. Furthermore, one can be observed that the decrease of $\alpha$ values lead to increase of the density of nutrient which makes the decrease of the necrotic cells faster. This also means that the numerical results are compatible biologically with the tumor growth that is the increase of the density of the nutrient leads to the decrease of the density of the necrotic cells. One can conclude finally that the quiescent cells are more likely to become the proliferative cells by the selected parameters.

In addition to these figures, from a mathematical angle, methods' estimation errors and their convergence rates are also listed in Table 1 and Table 2. The recorded errors are measured in the infinity norm. That is,

$E_{L_{\infty}}(p)=\max _{0 \leq i \leq N_{t}}\left|p_{i}-p^{r e f}\right|, \quad E_{L_{\infty}}(q)=\max _{0 \leq i \leq N_{t}}\left|q_{i}-q^{r e f}\right|, \quad E_{L_{\infty}}(n)=\max _{0 \leq i \leq N_{t}}\left|n_{i}-n^{r e f}\right|$ 
Table 1: Error estimation and order of convergence of RK4 for $p, q, n$

\begin{tabular}{|l|l|l|l|l|l|l|}
\hline$\Delta \boldsymbol{t}$ & \multicolumn{1}{|c|}{$\boldsymbol{E}_{\boldsymbol{L}_{\infty}}(\boldsymbol{p})$} & Order & \multicolumn{1}{|c|}{$\boldsymbol{E}_{\boldsymbol{L}_{\infty}}(\boldsymbol{q})$} & Order & $\boldsymbol{E}_{\boldsymbol{L}_{\infty}}(\boldsymbol{n})$ & Order \\
\hline 0.2 & $7.4095 \mathrm{e}-06$ & & $6.3409 \mathrm{e}-06$ & & $4.8696 \mathrm{e}-06$ & \\
\hline 0.1 & $4.9392 \mathrm{e}-07$ & 3.907 & $4.2219 \mathrm{e}-07$ & 3.9087 & $3.2289 \mathrm{e}-07$ & 3.9147 \\
\hline 0.05 & $3.1884 \mathrm{e}-08$ & 3.9534 & $2.7238 \mathrm{e}-08$ & 3.9542 & $2.0791 \mathrm{e}-08$ & 3.9570 \\
\hline 0.025 & $2.0252 \mathrm{e}-09$ & 3.9767 & $1.7297 \mathrm{e}-09$ & 3.9771 & $1.3190 \mathrm{e}-09$ & 3.9784 \\
\hline
\end{tabular}

Table 2: Error estimation and order of convergence of SSP-RK3 for p,q,n

\begin{tabular}{|l|l|l|l|l|l|l|}
\hline \multicolumn{1}{|c|}{$\Delta \boldsymbol{t}$} & \multicolumn{1}{|c|}{$\boldsymbol{E}_{\boldsymbol{L}_{\infty}}(\boldsymbol{p})$} & Order & \multicolumn{1}{|c|}{$\boldsymbol{E}_{\boldsymbol{L}_{\infty}}(\boldsymbol{q})$} & Order & $\boldsymbol{E}_{\boldsymbol{L}_{\infty}}(\boldsymbol{n})$ & Order \\
\hline 0.2 & $2.8918 \mathrm{e}-04$ & & $1.6349 \mathrm{e}-04$ & & $1.1337 \mathrm{e}-04$ & \\
\hline 0.1 & $3.8524 \mathrm{e}-05$ & 2.9082 & $2.1684 \mathrm{e}-05$ & 2.9145 & $1.2058 \mathrm{e}-05$ & 3.2330 \\
\hline 0.05 & $4.9716 \mathrm{e}-06$ & 2.9540 & $2.7924 \mathrm{e}-06$ & 2.9570 & $1.3730 \mathrm{e}-06$ & 3.1345 \\
\hline 0.025 & $6.3142 \mathrm{e}-07$ & 2.9770 & $3.5429 \mathrm{e}-07$ & 2.9785 & $1.6344 \mathrm{e}-07$ & 3.0706 \\
\hline
\end{tabular}

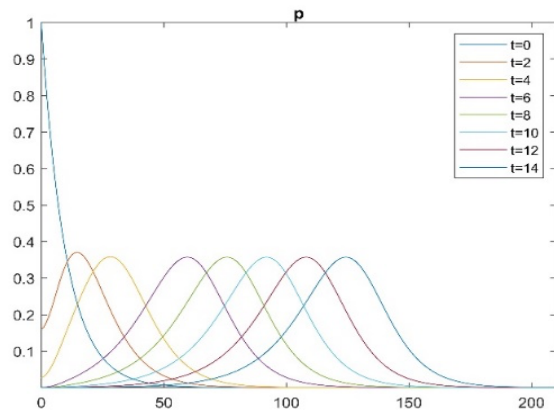

(a)

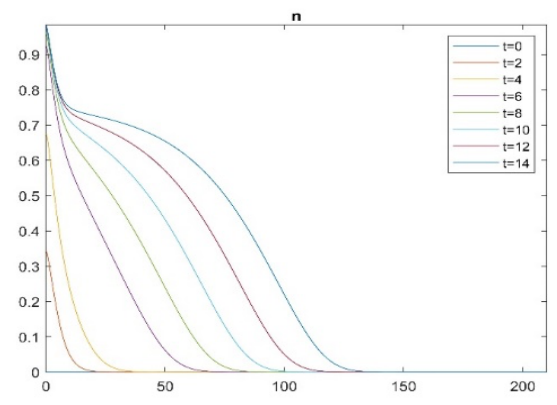

(c)

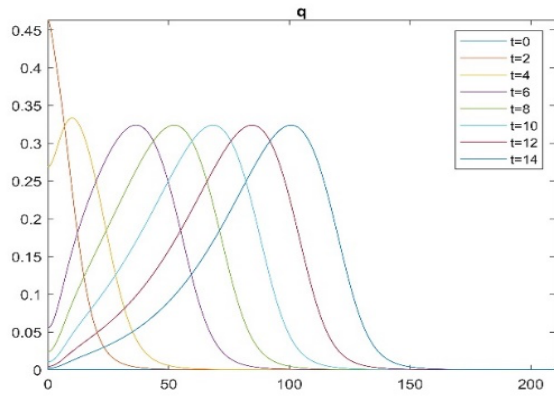

(b)

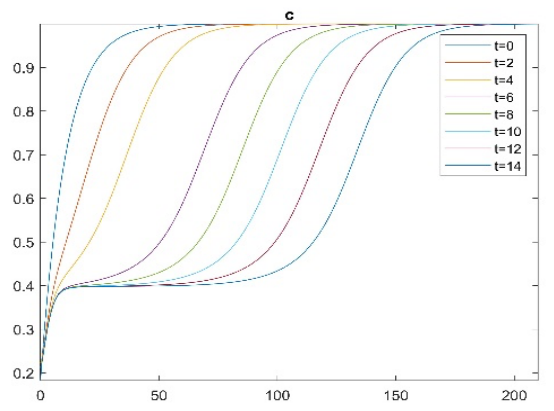

(d)

Figure 4. The densities of proliferating (a), quiescent (b), and the necrotic cell (c) for $\alpha=0.8$. The obtained results belong to SSP-RK3.

It is crucial to note that the main attention of this study is time discretization. Thus, $\mathrm{p}^{\wedge}$ ref, $\mathrm{q}^{\wedge}$ ref, and $\mathrm{n}^{\wedge}$ ref denote the reference solution where $\Delta t=0.001$. Here, Table 1 lists the errors and order of convergence of RK4 whereas Table 2 presents those values of SSPRK3.
As well as the biological compatibility, tables are also evidence that the performed methods are consistent with theoretical results given in the literature.

\section{CONCLUSION}

The main purpose of the study is to propose alternative numerical solution techniques for solving 


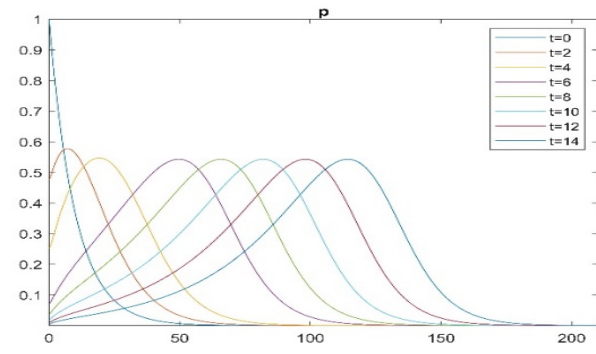

(a)

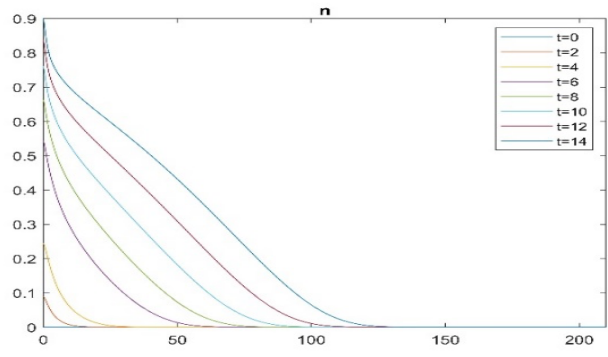

(c)

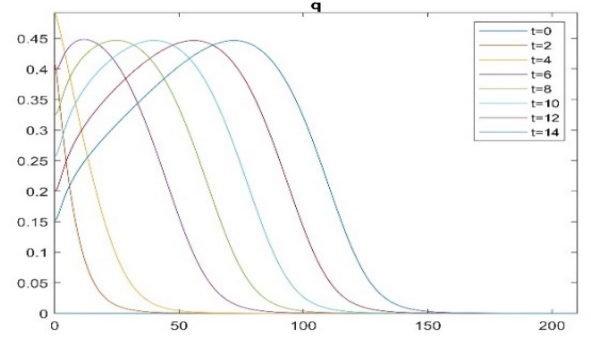

(b)

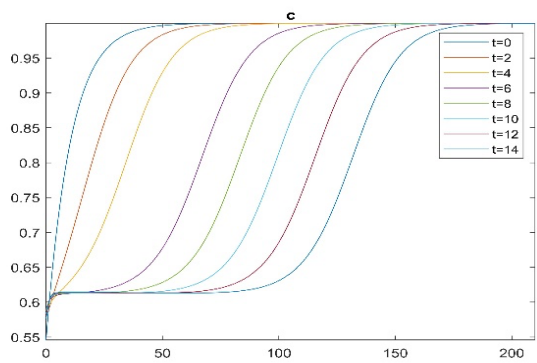

(d)

Figure 5. The densities of proliferating (a), quiescent (b), and the necrotic cell (c) for $\alpha=0.4$. The obtained results belong to SSP-RK3.

the avascular tumor growth model which contains the proliferative, quiescent, and necrotic zone. This is not to say the study is just mathematical, on the contrary, it presents also biological perspectives. To do so, Sherratt-Chaplin's avascular model has been considered and has been solved numerically. For solving the model, the discretization of the spatial domain has been fixed to the central difference approximation whereas the temporal domain has been integrated via two different techniques such that SSP-RK3 and RK4. Both numerical schemes have been tested on the mathematical model. For a reliable comparison, the parameters of the model are chosen in line with the literature. All indicated figures have shown that both methods have recorded similar results to those of the literature. Moreover, as stated in the discussion part that both methods are perfectly compatible with the biological truth. Furthermore, the numerical results are also enriched with the tables which list the errors and the rate of convergence of the numerical method, computationally. Finally, in the light of the knowledge of all valuable aforementioned findings and discussions, one can be concluded that both proposed methods can be considered as the alternative methods for solving such problems.

Acknowledgement: The authors would like to thank both the Editor in Chief and the referees for their valuable time and consideration.
Conflict of interests: The authors declare that they have no competing interests.

Grant \& Fundings: The authors declare that there is no received financial support for this research.

Peer-review: Externally peer-reviewed.

\section{REFERENCES}

1. Byrne HM, Alarcon T, Owen MR, Webb SD and Maini PK. Modelling aspects of cancer dynamics: a Review. Phil. Trans. R. Soc. A. 2006; 364;1563-1578.

2. Jiang Y, Pjesivac-Grbovic J, Cantrell C, Freyer JP. A multiscale model for avascular tumor growth. Biophysical Journal 2005; 89(6); 38843894.

3. Bagherpoorfard M, Soheili A. A numerical method based on the moving mesh for the solving of a mathematical model of the avascular tumor growth. Computational Methods for Differential Equations 2021; 9(2): 327-346.

4. Kunz-Schughart LA, Kreutz $M$, Knuechel R. Multicellular spheroids: a three-dimensional in vitro culture system to study tumour biology. International Journal of Experimental Pathology, 1998; 79: 1-23.

5. Thomlinson RH, Gray LH. The histological structure of some human lung cancers and possible implications for radiotherapy. $\mathrm{Br} J$ Cancer 1955; 9: 539-549. 
6. Burton AC. Rate of growth of solid tumours as problem of diffusion. Growth 1966; 30: 157-176.

7. Greenspan HP: Models for the growth of a solid tumor by diffusion. Stud Appl Math 1972; 52: 317 340.

8. Chaplin MA, Benson DL, Maini PK. Nonlinear diffusion of a growth inhibitory factor in multicell spheroids. Math. Biosci. 1994; 121(1): 1-13.

9. Byrne HM, Chaplin MAJ. Growth of necrotic tumors in the presence and absence of inhibitors. Math. Biosci. 1996; 135(2): 187-216.

10. Ward JP, King JR. Mathematical modelling of avascular-tumour growth II: modelling growth saturation. Math. Med. Biol. 1997; 14(1): 39-69.

11. Sherratt JA, Chaplin MA. A new mathematical model for avascular tumor growth. J. Math. Biol. 2001; 43(4): 291-312.

12. Unsal S, Acar A, Itik M, et al. Personalized Tumor Growth Prediction Using Multiscale Modeling. Journal of Basic and Clinical Health Sciences 2020; 4(3), 347-363.

13. Roose T, Chapman S, Maini P. Mathematical Models of Avascular Tumor Growth. SIAM Review 2007; 49(2): 179-208.

14. Kuang $Y$, Nagy JD, Eikenberry SE. Introduction to Mathematical Oncology (Chapman \& Hall/CRC Mathematical and Computational Biology), 1st ed. CRC Press; 2016.

15. Mahmood MS, Mahmood S, Dobrota D. A numerical algorithm for avascular tumor growth model. Mathematics and Computers in Simulation 2010; 80(6): 1269-1277.

16. Said NM, Ibrahim A, Alias N, Numerical Simulation of Hypoxic Cell Regulation in Avascular Tumor Growth. AIP Conference Proceedings 2013; 1522: 400-404.

17. Darbyshire PM. A System of Coupled Nonlinear Partial Differential Equations Describing Avascular Tumour Growth Are Solved Numerically Using Parallel Programming to Assess Computational Speedup. Computational Biology and Bioinformatics. 2015; 3(5): 65-73.

18. Amoddeo A. Modeling Avascular Tumor Growth: Approach with an Adaptive Grid Numerical Technique. Journal of Multiscale Modelling 2018; 9(3): $\quad$ Article Id: 1840002. http://dx.doi.org/10.1142/S1756973718400024

19. Bagherpoorfard $M$, Soheili AR. A numerical method based on the moving mesh for the solving of a mathematical model of the avascular tumor growth. Computational methods for Differential Equations 2021; 9(2): 327-346.

20. Ali A, Hussain M, Ghaffar A et al. Numerical simulations and analysis for mathematical model of avascular tumor growth using Gompertz growth rate function. Alexandria Engineering Journal 2021; 60(4): 3731-3740.

21. Durran DR. Numerical Methods for Fluid Dynamics. 2nd ed. New York, NY: Springer; 2010. 